\title{
Dual regulatory pathways of flagellar gene expression by ClpXP protease in enterohaemorrhagic Escherichia coli
}

\author{
Ryo Kitagawa, † Akiko Takayat and Tomoko Yamamoto
}

Correspondence

Tomoko Yamamoto

tomoko-y@p.chiba-u.ac.jp

Received 7 May 2011

Revised 20 August 2011

Accepted 1 September 2011

\author{
Department of Microbiology and Molecular Genetics, Graduate School of Pharmaceutical Sciences, \\ Chiba University, Chiba 263-8522, Japan
}

\begin{abstract}
In enterobacteria such as Escherichia coli and Salmonella species, flagellar biogenesis is strictly dependent upon the master regulator flhDC. Here, we demonstrate that in enterohaemorrhagic E. coli (EHEC), the flagellar regulon is controlled by ClpXP, a member of the ATP-dependent protease family, through two pathways: (i) post-translational control of the FlhD/FlhC master regulator and (ii) transcriptional control of the flhDC operon. Both FlhD and FlhC proteins accumulated markedly following ClpXP depletion, and their half-lives were significantly longer in the mutant cells, suggesting that ClpXP is responsible for degrading FlhD and FlhC proteins, leading to downregulation of flagellar expression. ClpXP was involved in regulating the transcription of the $f / h D$ promoter only when the cells had entered stationary phase in a culture medium that markedly induced expression of the locus of enterocyte effacement (LEE).

Comparative analyses of transcription from the fIhD promoter in EHEC cells with different genetic backgrounds suggested that the downregulation of $f / h D C$ expression by ClpXP is dependent on the LEE-encoded GrIR-GrlA system. We have also shown that the degradation of FlhD and FlhC by ClpXP is responsible for downregulating flagellar expression even when LEE expression is induced.
\end{abstract}

\section{INTRODUCTION}

Enterohaemorrhagic Escherichia coli (EHEC) O157:H7 is a causative agent of bloody diarrhoea, non-bloody diarrhoea and haemolytic uraemic syndrome in humans (Akashi et al., 1994). EHEC colonizes the intestine and causes a destructive lesion of the intestinal enterocyte, referred to as the attaching and effacing $(\mathrm{A} / \mathrm{E})$ lesion. The genes responsible for the formation of $\mathrm{A} / \mathrm{E}$ lesions are located on a pathogenicity island called the locus of enterocyte effacement (LEE). The LEE genes are organized into five operons which are under strict control (Friedberg et al., 1999). Three regulators, Ler, GrlR and GrlA, encoded in the LEE have been well characterized: Ler is a central activator of the transcription of most LEE genes, and GrlA and GrlR are positive and negative regulators, respectively, for ler transcription (Iyoda et al., 2006). In addition to the control by those regulatory elements encoded in the LEE, various regulatory elements encoded outside the locus, such as BipA (Grant et al., 2003), IHF (Friedberg et al., 1999), H-NS (Bustamante et al., 2001; Umanski et al., 2002) and QseA (Sperandio et al., 2002), are

†These authors contributed equally to this work.

Abbreviations: EHEC, enterohaemorrhagic Escherichia coli; LEE, locus of enterocyte effacement.

A supplementary table of primer sequences is available with the online version of this paper. involved in the control of LEE expression. We have previously reported that the ATP-dependent protease, ClpXP, is a positive regulator of LEE expression (Tomoyasu et al., 2005). ClpXP protease is composed of a ClpP proteolytic component and a ClpX component that binds substrate proteins, denatures them and translocates the unfolded polypeptides into the ClpP degradation chamber (Ortega et al., 2000). ClpXP is a bipartite protease responsible for degrading certain key regulatory proteins such as RpoS, which is a stationary-phase-specific sigma factor for RNA polymerase, and aberrant translation products bearing the SsrA degradation tag, added co-translationally to nascent polypeptides when ribosomes stall (Gottesman et al., 1998; Zhou et al., 2001). Our previous studies demonstrated that positive regulation of LEE expression by ClpXP is via two pathways, namely the RpoS-dependent and RpoS-independent pathways (Tomoyasu et al., 2005). ClpXP is involved in the expression of LEE at the control of the levels of the negative regulator GrlR in EHEC which could be the RpoS-independent pathway (Iyoda \& Watanabe, 2005).

In the course of phenotypic characterization of a ClpXPdepleted mutant derived from EHEC O157:H7 Sakai, we have found that depletion of ClpXP results in overproduction of FliC, a major component of the flagellar filament, whereas there is a marked decrease in LEE-related proteins. The flagellum is an organelle consisting of three 
distinctive structural parts, the basal body consisting of a central rod and several rings, the short curved structure called the hook, and the long helical filament which consists of an assembly of $\sim 20000$ subunits of a single protein, FliC (Macnab, 2003). Over 40 genes are specifically required for the flagellar formation and function in E. coli, and these are organized into a transcriptional hierarchy that underlies temporal and spatial control of the biogenesis program (Macnab, 1996). At the apex of the hierarchy is the class 1 flhDC operon. The two operon products, FlhD and FlhC, form a hetero-oligomer to activate the $\sigma^{70}$ promoter of class 2 genes that encode hook-basal body proteins, as well as the alternate sigma factor, FliA. FliA transcribes class 3 genes involved in motor, chemotaxis and filament structures. Since the FlhD/FlhC is essential for transcription of all the genes in the flagellar cascade, it is also called a master regulator. Transcription of the flhDC operon is known to be regulated by a number of global regulatory signals in E. coli and related bacteria (Iyoda et al., 2006; Shi et al., 1993; Soutourina et al., 2002). In addition, the $\mathrm{FlhD} / \mathrm{FlhC}$ is modulated at the post-transcriptional and post-translational levels (Tomoyasu et al., 2003; Wozniak et al., 2009).

Here we provide evidence showing that ClpXP protease tightly regulates the flagellar expression by degrading the FlhD/FlhC master regulator in EHEC. We propose that the flagellar regulon in EHEC is controlled by ClpXP protease through two pathways, namely post-translational control of the FlhD/FlhC master regulator by degrading them and transcriptional control of the flhDC operon through the LEE-encoded GrlR-GrlA regulatory system.

\section{METHODS}

Bacterial strains and growth conditions. Bacterial strains and plasmids used in this study are shown in Table 1. Unless otherwise specified, bacteria were grown in L-broth (1\% Bacto tryptone, $0.5 \%$ Bacto yeast extract, $0.5 \%$ sodium chloride, $\mathrm{pH}$ 7.4) or L-agar.

Construction of plasmids pTKY802 and pTKY753. Plasmid pTKY802, in which the expression of $f l h D C$ operon is controlled by the $\mathrm{P}_{\mathrm{AllacO}-1}$ promoter, was constructed by amplifying a BamHIHindIII fragment carrying the flhDC operon by PCR with flhDCBamHI-F and flhDC-HindIII-R primers (Supplementary Table S1, available with the online version of this paper) and subsequent cloning of the fragment into pUHE21-2 $\mathrm{fd} 12$ cleaved with BamHI and HindIII. In plasmid pTKY753 containing the fliA promoter, the locus was amplified by PCR with the primers fliApro-HindIII-F and fliApro-BamHI-R (Supplementary Table S1). The $320 \mathrm{bp}$ fragment generated was cleaved with BamHI at the $5^{\prime}$ end and HindIII at the $3^{\prime}$ end before being cloned into the vector pCB192 cleaved with BamHI and HindIII.

Construction of $\Delta f \mid h D C, \Delta g r l R$ and $\Delta g r l A$ mutants, and flhDlacZ fusion strain. To construct the $\Delta$ flhDC mutants from $\mathrm{O} 157: \mathrm{H} 7$ Sakai strain, the DNA fragment in the $f l h D C$ region was initially amplified by PCR from the chromosome of strain CS5295, using the primers flhDC-BamHI-F and flhDC-HindIII-R (Supplementary Table S1). The 1067 bp fragment was cleaved with BamHI and HindIII, and cloned into the vector pHSG399. The resulting plasmid was digested with HincII to eliminate the $600 \mathrm{bp}$ central fragment of $f l h D C$ and was self-ligated. The resultant plasmid was cleaved at the HindIII site and the overhanging end was filled in with T4 DNA polymerase, and then cleaved at the SacI site. The generated fragment was ligated to the suicide vector pCVD442 cleaved with SmaI and SacI. A sucide feature of pCVD442 is based on one of the replication origins of R6K that becomes functional only when the $\pi$ protein is encoded by the pir gene. The resultant mutator plasmid, pTKY883, was introduced into strain SM10 $\lambda$ pir, which can mobilize the plasmid by the conjugative function provided in trans. Conjugative crosses with strain CS5295 $\left(c l p P X^{+}\right)$or CS5297 $(\Delta c l p P X)$ were carried out. The chromosomal flhDC operon was replaced by the disrupted $f h D C$ operon on the suicide vector by double recombination. The destruction of the $f h D C$ operon in the resulting strains, CS5835 or CS5836, was verified by amplification of the chromosomal DNA by PCR and Southern blot analysis. To measure the levels of transcription from flhD promoter, strains carrying a transcriptional $f l h D$-lac $Z$ fusion at the lac locus on the chromosome were constructed. A fragment carrying $f l h D$ promoter was amplified by PCR with the primers flh $\Delta$ pro-HindIII-F and flh $\Delta$ pro-BamHI-R (Supplementary Table S1) from CS5295 chromosomal DNA, and the PCR product was ligated upstream of the lac $Z$ gene in plasmid pZA3lacIZ. The resultant plasmid, pTKY884 was cleaved with SacI and SacII, and the overhanging end was blunted with T4 DNA polymerase. The generated fragment was ligated to the suicide vector pCVD442 cleaved with SmaI. The resulting plasmid, pTKY885 was introduced

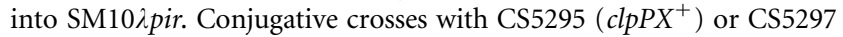
$(\triangle c l p P X)$ were then carried out. The resultant strains, CS6181 and CS6183, carry the $f l h D$ promoter-lacZ fusion on the chromosome with the disrupted lacI gene. Allelic exchange was checked by Southern blot analysis and PCR amplification of the chromosomal DNA with lacI-F and lacZ-R (Supplementary Table S1). To construct a series of O157:H7 Sakai strains with $g r l R$ and $g r l A$ mutations, the one-step method of chromosomal gene inactivation with $\lambda$ Red recombinase was used. PCR products containing kanamycin-resistance cassettes flanked by 45 or $50 \mathrm{bp}$ of homology to the $5^{\prime}$ and $3^{\prime}$ termini of $\mathrm{grlR}$ or $\mathrm{grlA}$ genes were electroporated into competent cells of parental strains carrying plasmid pKD46 encoding the $\lambda$ Red gene. To construct strain CS6270 and CS6271, PCR fragments were amplified from the pKD4 encoding kanamycin-resistance cassette, using primers $g r l R-\lambda$ red-F and grlR- $\lambda$ red-R (Supplementary Table S1). To construct strain CS6272 and CS6273, primers grlA- $\lambda$ red-F and grlA- $\lambda$ red-R (Supplementary Table S1) were used to amplify the fragment from pKD4. Kanamycinresistant colonies were selected and the resultant clones were streaked at $37{ }^{\circ} \mathrm{C}$ to isolate those that had lost pKD46. PCR and Southern blot analysis confirmed mutation of the grlR or grlA gene. The FRT-flanked kanamycin cassette was removed after transformation with pCP20, as previously described (Datsenko \& Wanner, 2000).

SDS-PAGE and immunoblotting analysis. Gel electrophoresis and immunoblotting analysis were carried out according to previously described methods (Takaya et al., 2006).

\section{RESULTS}

\section{ClpXP protease is responsible for the turnover of the FIhD/FIhC master regulator in EHEC}

In a previous study, we constructed a $\triangle c l p P X$ mutant from O157:H7 Sakai by removing the central region of the clpPX operon, followed by insertion of a chloramphenicol resistance cassette (Tomoyasu et al., 2005). The insertion did not block the expression of the downstream gene, lon, by its own promoter. Fig. 1(a) shows that FlhD and FlhC proteins accumulate in EHEC cells when the clpPX operon is disrupted, indicating that ClpXP protease negatively 
Table 1. Bacterial strains and plasmids used in this study

$\mathrm{NA}$, nalidixic acid resistance; $\mathrm{Km}$, kanamycin resistance; $\mathrm{Cm}$, chloramphenicol resistance.

\begin{tabular}{|c|c|c|}
\hline Strain or plasmid & Relevant characteristics & Reference or source \\
\hline \multicolumn{3}{|l|}{ E. coli } \\
\hline SM10גpir & 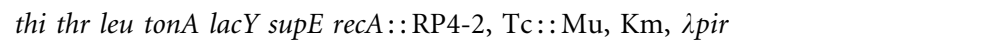 & Laboratory collection \\
\hline O157: H7 Sakai & E. coli O157: H7 derived from Sakai outbreak (RIMD 0509952) & Akashi et al. (1994) \\
\hline CS5295 & O157: H7 Sakai NA & Tomoyasu et al. (2005) \\
\hline CS5297 & CS5295 clpPX:: Cm & Tomoyasu et al. (2005) \\
\hline CS5693 & CS5295/pTKY753 & This study \\
\hline CS5698 & CS5297/pTKY753 & This study \\
\hline CS5829 & CS5295/pZA4 lacI ${ }^{q}$ and pTKY802 & This study \\
\hline CS5830 & CS5297/pZA4 lacI ${ }^{q}$ and pTKY802 & This study \\
\hline CS5835 & CS5295 $\Delta f l h D C$ & This study \\
\hline CS5836 & CS5297 $\Delta f h D C$ & This study \\
\hline CS6181 & $\begin{array}{l}\text { CS5295 } \Delta \text { lacI } 820 \mathrm{bp} \text { fragment containing flhD promoter-lacZ } \\
\text { transcriptional fusion on chromosome }\end{array}$ & This study \\
\hline CS6183 & $\begin{array}{l}\text { CS5297 } \Delta \text { lacI } 820 \text { bp fragment containing flhD promoter-lacZ } \\
\text { transcriptional fusion on chromosome }\end{array}$ & This study \\
\hline CS6229 & CS5835/pTKY753 & This study \\
\hline CS6230 & CS5836/pTKY753 & This study \\
\hline CS6292 & CS5835/pZA4lacI ${ }^{q}$ and pTKY802 & This study \\
\hline CS6293 & CS5836/pZA4lacI $I^{q}$ and pTKY802 & This study \\
\hline CS6270 & CS5295 grlR::FRT & This study \\
\hline CS6271 & CS5297 grlR: :FRT & This study \\
\hline CS6272 & CS5295 grlA:: FRT & This study \\
\hline CS6273 & CS5297 grlA:: FRT & This study \\
\hline CS6281 & CS6270/pTKY753 & This study \\
\hline CS6282 & CS6271/pTKY753 & This study \\
\hline CS6283 & CS6272/pTKY753 & This study \\
\hline CS6284 & CS6273/pTKY753 & This study \\
\hline CS6320 & CS6181 grlR::FRT & This study \\
\hline CS6321 & CS6183 grlR::FRT & This study \\
\hline CS6322 & CS6181 grlA:: FRT & This study \\
\hline CS6323 & CS6183 grlA:: FRT & This study \\
\hline \multicolumn{3}{|l|}{ Plasmids } \\
\hline pUHE21-2 2 fd 12 & $\mathrm{P}_{\mathrm{AllacO}-1}$ system vector & Gamer et al. (1992) \\
\hline pZA4lac $I^{q}$ & $\operatorname{lacI}^{q}$ & Laboratory collection \\
\hline pZA3lacIZ & Carrying fragments of lacI and lacZ & Laboratory collection \\
\hline pHSG399 & Cloning vector & Laboratory collection \\
\hline pCB192 & Promoter cloning vector & Schneider \& Beck (1986) \\
\hline pKD4 & Carrying kanamycin-resistance gene & Datsenko \& Wanner (2000) \\
\hline pKD46 & $\lambda \operatorname{Red}(\gamma, \beta$, exo $)$ & Datsenko \& Wanner (2000) \\
\hline pCP20 & Carrying the yeast Flp recombinase gene, $f l p$ & Cherepanov \& Wackernagel (1995) \\
\hline pCVD442 & Carrying oriy of R6K, mobRP4, bla, sacB & Donnenberg \& Kaper (1991) \\
\hline pTKY753 & pCB192 with $320 \mathrm{bp}$ fragment containing fliA promoter & This study \\
\hline pTKY802 & pUHE21-2 $\Delta$ fd12 carrying $f l h D C$ operon & This study \\
\hline pTKY883 & pCVD442 with flhDC operon disrupted by HincII digestion & This study \\
\hline pTKY884 & Carrying fragments of lacI, lac $Z$ and $820 \mathrm{bp}$ fragment containing $f l h D$ promoter & This study \\
\hline pTKY885 & pCVD442 with SacI-SacII fragment containing lacI, lacZ and flhD promoter & This study \\
\hline
\end{tabular}

controls the cellular levels of the FlhD/FlhC master regulator for flagellar gene expression. To examine whether ClpXP controls the turnover of FlhD and FlhC proteins, leading to the negative regulation of flagellar gene expression in EHEC, we determined the in vivo half-lives of these two proteins. Expression of the flhDC operon was initiated from the $\mathrm{P}_{\text {AllacO-1 }}$ promoter on plasmid pTKY802 in a $\triangle f l h D C$ background on the chromosome by adding IPTG. Tetracycline was added to prevent de novo synthesis of proteins and the cellular levels of FlhD and FlhC at the indicated times after tetracycline addition are shown in Fig. 1(b). Whereas FlhD and FlhC decayed with half-lives of 


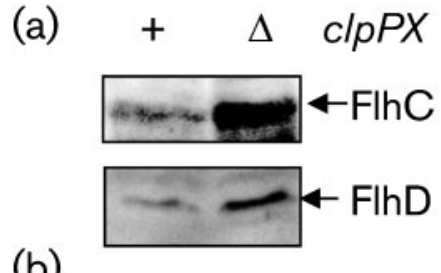

(b)
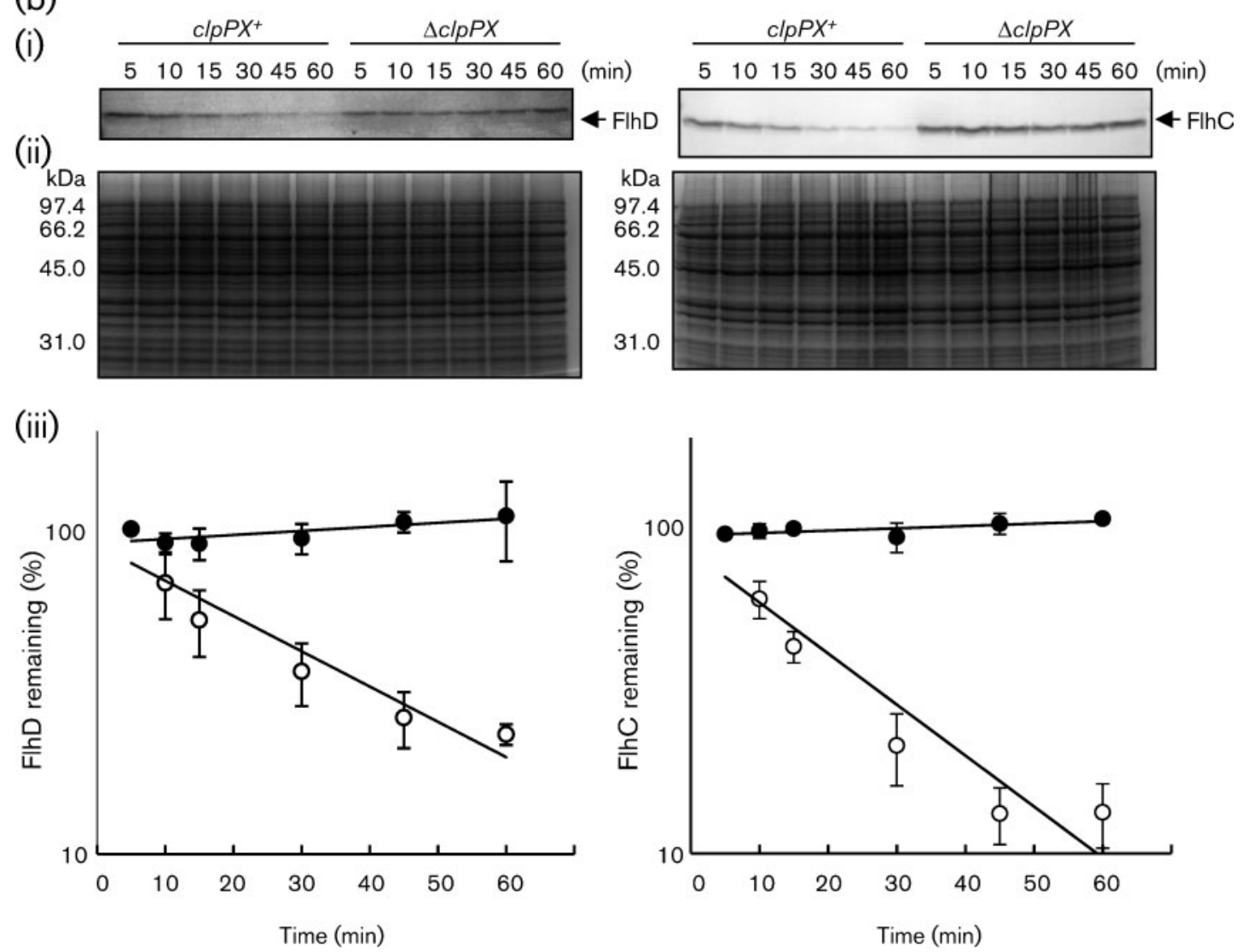

Fig. 1. Cellular contents (a) and in vivo stabilities (b) of FlhC and FlhD proteins in EHEC cells with $c / p P X^{+}$or $\triangle c / p P X$ background. (a) Whole-cell extracts were prepared from strains CS5295 (clpPX $\left.{ }^{+}\right)$and CS5297 $(\triangle c / p P X)$, and then separated by using SDS-PAGE. The separated proteins were immunostained with anti-FlhC or anti-FlhD antibodies. (b) Strain CS6292 $\left(c / p P X^{+}\right)$and CS6293 $(\Delta c / p P X)$ were grown to $\mathrm{OD}_{600} 0.5$ at $37{ }^{\circ} \mathrm{C}$ in L-broth containing $20 \mu \mathrm{M}$ IPTG to induce flhDC expression for $1 \mathrm{~h}$. Cultures were washed once with fresh medium, followed by medium containing tetracycline $\left(100 \mu \mathrm{g} \mathrm{ml} \mathrm{l}^{-1}\right)$ and glucose $(2 \%)$ to block translation and transcription of flhDC. Aliquots of cells were taken at the indicated times and mixed with pre-chilled TCA (final concentration, $10 \%$ ). (i) The proteins were separated by using $15 \%$ (for FlhC) or $17 \%$ (for FlhD) SDS-PAGE, before being immunostained with anti-FlhC antibody or anti-FlhD antibody. (ii) Coomassie blue-stained gel patterns of the same samples used for immunoblotting. (iii) Quantification of the precipitated proteins in $c / p P X^{+}(\bigcirc)$ and $\triangle c / p P X(\bullet)$ cells using Quantity One (BioRad) relative to the values at $5 \mathrm{~min}$. Mean \pm SD values of at least three independent experiments are shown.

$27.7 \mathrm{~min}$ and $13.8 \mathrm{~min}$, respectively, in $c l p P X^{+}$cells after the arrest of de novo synthesis, they had not decayed in the $\triangle c l p P X$ cells after a $60 \mathrm{~min}$ chase, indicating that they were stabilized after depletion of ClpXP. These results suggest that ClpXP could be involved in the turnover of FlhD and FlhC.

To determine whether ClpXP regulates the expression of the flagellar regulon only by the FlhD/FlhC-dependent pathway, the effect of $c l p P X$ disruption on the expression of
fliA, which belongs to the class 2 genes, was examined using lac $Z$ fusion strains with a $f l h D C^{+}$or $\triangle f l h D C$ background on the chromosome. Whereas disruption of the clpPX operon increased fliA transcription $\sim 2.5$-fold in $f h D C^{+}$cells, the stimulation of fliA transcription in $\triangle c l p P X$ cells was completely suppressed by the $\triangle$ flhDC mutation (Fig. 2). Since the fliA product, $\sigma^{28}$, is responsible for transcribing all class 3 genes, ClpXP could control the expression of the flagellar regulon through this single pathway. 


\section{ClpXP protease controls the transcription of the flhDC operon in EHEC under conditions in which LEE expression is induced}

To determine whether ClpXP is involved in the transcriptional control of $f l h D C$, we examined the levels of transcription of the flhDC operon in EHEC strains with a $c l p P X^{+}$or $\triangle c l p P X$ background. EHEC cultures were also grown in L-broth and a tissue culture medium such as Dulbecco's modified Eagle's medium (DMEM), in which LEE genes are known to be strongly expressed (Abe et al., 2002), and the efficiencies of transcription of the flhDC operon were compared.

The overnight EHEC cultures grown in L-broth were diluted 1:100 with fresh L-broth or DMEM and incubated at $37{ }^{\circ} \mathrm{C}$. The cells were grown to exponential phase $\left(\mathrm{OD}_{600}\right.$ $0.5)$ or stationary phase ( $16 \mathrm{~h}$ after initiation of growth). The levels of transcription of the flhDC operon in cells with the $\triangle c l p P X$ background were compared with $c l p P X^{+}$cells by measuring the activities of the flhD-lac $Z$ transcriptional fusion. In $c l p P X^{+}$cells grown to exponential phase, $f l h D-$ lacZ fusion activity was lower in DMEM than in L-broth (Fig. 3a). When the cultures had entered stationary phase, the $f l h D-l a c Z$ fusion activity in cells grown in DMEM was also lower than in cells grown in L-broth, but disruption of the $\operatorname{clpPX}$ operon increased the $f l h D-l a c Z$ activity to the same levels seen in L-broth (Fig. 3b). In contrast, the increase following disruption of $c l p P X$ did not occur in exponential phase cultures grown in DMEM (Fig. 3a). The results also showed that when cells were grown in L-broth,

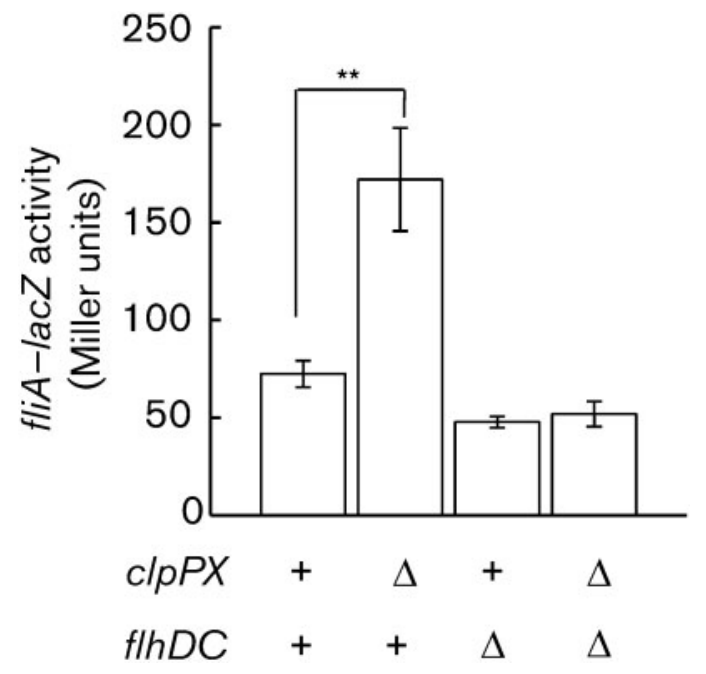

Fig. 2. Effect of depletion of ClpXP protease on the expression of the transcriptional fliA-lacZ fusion in the absence of $f / h D C$ operon in EHEC cells. The levels of $\beta$-galactosidase activity of bacterial cells of strains CS5693 (clpPX $X^{+}$flhDC $\left.{ }^{+}\right)$, CS5698 $(\Delta c / p P X$ $\left.f l h D C^{+}\right), \operatorname{CS} 6229\left(c / p P X^{+} \Delta f / h D C\right)$ and CS6230 ( $\Delta c / p P X$ $\triangle f l h D C$ ) were assayed according to the previously described method (Takaya et al., 2006). The enzyme units are mean \pm SD of at least three independent assays. ${ }^{\star \star} P<0.001$.
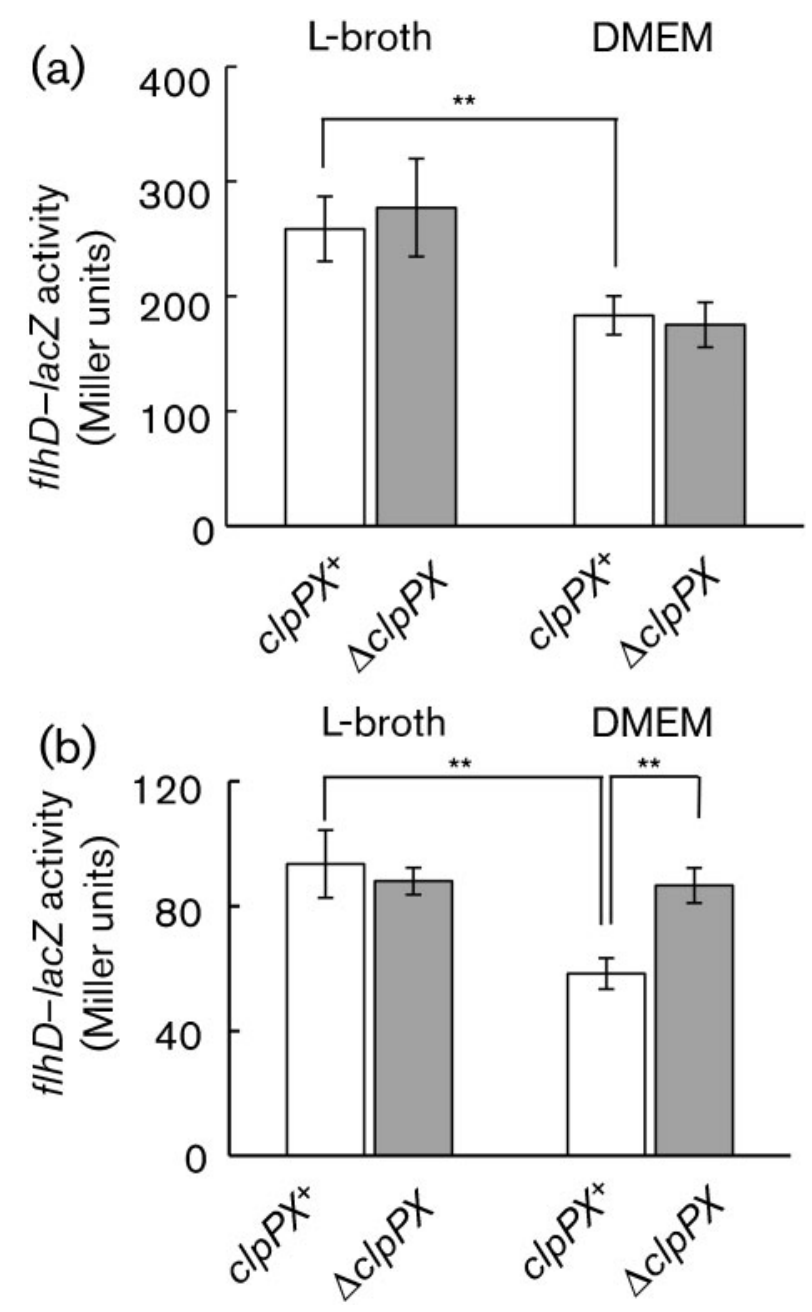

Fig. 3. Effect of depletion of ClpXP protease on the expression of transcriptional flhD-lacZ fusion in the EHEC cells grown under various growth conditions. The activities of $f / h D$ promoter-lac $Z$ fusion in EHEC cells of strains CS6181 (clpPX ${ }^{+}$; white bars) and CS6183 ( $\triangle c / p P X$; grey bars) were assayed. Bacterial cells were grown in L-broth or DMEM to $\mathrm{OD}_{600} 0.5$ (a; exponential phase) or for $16 \mathrm{~h}$ (b; stationary phase) at $37{ }^{\circ} \mathrm{C}$. The values represent the mean \pm SD of samples tested in triplicate. ${ }^{\star \star} P<0.001$.

clpPX disruption did not affect the transcription of the $f l h D C$ operon in either exponential or stationary phase. These results suggest that ClpXP is responsible for controlling $f h D C$ expression at the transcriptional level in bacterial cells specifically grown in DMEM and when they enter the stationary phase, i.e. conditions under which LEE expression is induced.

\section{Regulation of flagellar gene expression by ClpXP protease via dual pathways}

Expression of the flagellar regulon in E. coli has been shown to be under the control of various regulatory elements, including CRP and H-NS (Soutourina et al., 1999), OmpR 
(Shin \& Park, 1995), CsrA (Wei et al., 2001), IHF (YonaNadler et al., 2003), RcsB (Francez-Charlot et al., 2003), LrhA (Lehnen et al., 2002), QseD (Habdas et al., 2010), CadA (Vazquez-Juarez et al., 2008) and GrlA (Iyoda et al., 2006). Among these, GrlA acts as a negative regulator for transcription of the flhDC operon, but the mechanism remains unknown (Iyoda et al., 2006). GrlA, which was originally identified as an activator of LEE operon expression, is antagonized by GrlR. The levels of GrlR are regulated in a growth-phase-dependent manner; GrlR levels are minimal when EHEC cells enter stationary phase, thereby facilitating expression of the LEE operon (Iyoda \& Watanabe, 2005). To define the role of the GrlR-GrlA system in the control of flagellar expression by ClpXP, we measured the flhD-lac $Z$ fusion activities in EHEC cells with $\triangle c l p P X, \Delta g r l R$ and/or $\triangle g r l A$ mutations as genetic backgrounds when they had entered stationary phase in DMEM. clpPX disruption increased the $f l h D-l a c Z$ activity by 1.8 -fold (Fig. 4a; compare $c l p P X^{+}$and $\triangle c l p P X$ in a $g_{l l}{ }^{+} g r l A^{+}$ background). In contrast, disruption of $g r l R$ diminished the effect that $c l p P X$ disruption had on increasing the $f l h D-l a c Z$ activity; the $\operatorname{clp} P X$ disruption increased activity by 1.2 -fold in a $\Delta g r l R$ background (Fig. 4a; compare $c l p P X^{+}$and $\triangle c l p P X$ in a $\triangle g r l R$ background). Furthermore, disruption of grlA abolished the increase due to $c l p P X$ disruption (Fig. 4a; compare $c l p P X^{+}$and $\triangle c l p P X$ in a $\triangle g r l A$ background). Therefore, the regulation of transcription of the flhDC operon by ClpXP could be through the GrlR-GrlA pathway. When the $f l h D-l a c Z$ activity was compared in the $\operatorname{clp} P X^{+}$ background, disruption of $g r l R$ decreased $f l h D$ transcription moderately (Fig. 4a; see $g r l R^{+}$and $\Delta g r l R$ in a $c l p P X^{+}$ background). On the other hand, disruption of $\mathrm{grl}$ had no effect on the efficiency of transcription from the $f h D C$ operon promoter in the $c l p P X^{+}$background (Fig. 4a; see grlA ${ }^{+}$and $\triangle g r l A$ in a $c l p P X^{+}$background). This is similar to the results described in the previous report, in which the negative effect of grlA on $f l h D$ transcription could be shown only in cells overexpressing plasmid grlA (Iyoda et al., 2006). In contrast, when GrlA, reputedly a negative regulator for $f l h D$ transcription was depleted in the $\triangle c l p P X$ background, the $f l h D-l a c Z$ activity significantly decreased (Fig. 4a; see $g_{r l} A^{+}$and $\triangle g r l A$ in a $\triangle c l p P X$ background). This suggests that an additional factor(s) for regulating the transcription of the flhDC operon would be included in the GrlR-GrlA pathway.

We also monitored the transcriptional regulating activity of the FlhD/FlhC complex in EHEC cells grown under different conditions by measuring the efficiencies of the fliA promoter in cells with different genetic backgrounds. Fig. 4(b) shows the activities of the fliA-lac Z transcriptional fusion in EHEC cultures that had entered stationary phase in DMEM. Disruption of clpPX strongly increased transcription by the fliA promoter; the activity of the fliAlac $Z$ transcriptional fusion was increased fivefold (Fig. 4b; see $c l p P X^{+}$and $\triangle c l p P X$ in a $g r l R^{+} g r l A^{+}$background). Although grlR or grlA disruption mostly abolished the increase of $f l h D C$ transcription by $c l p P X$ disruption (Fig.
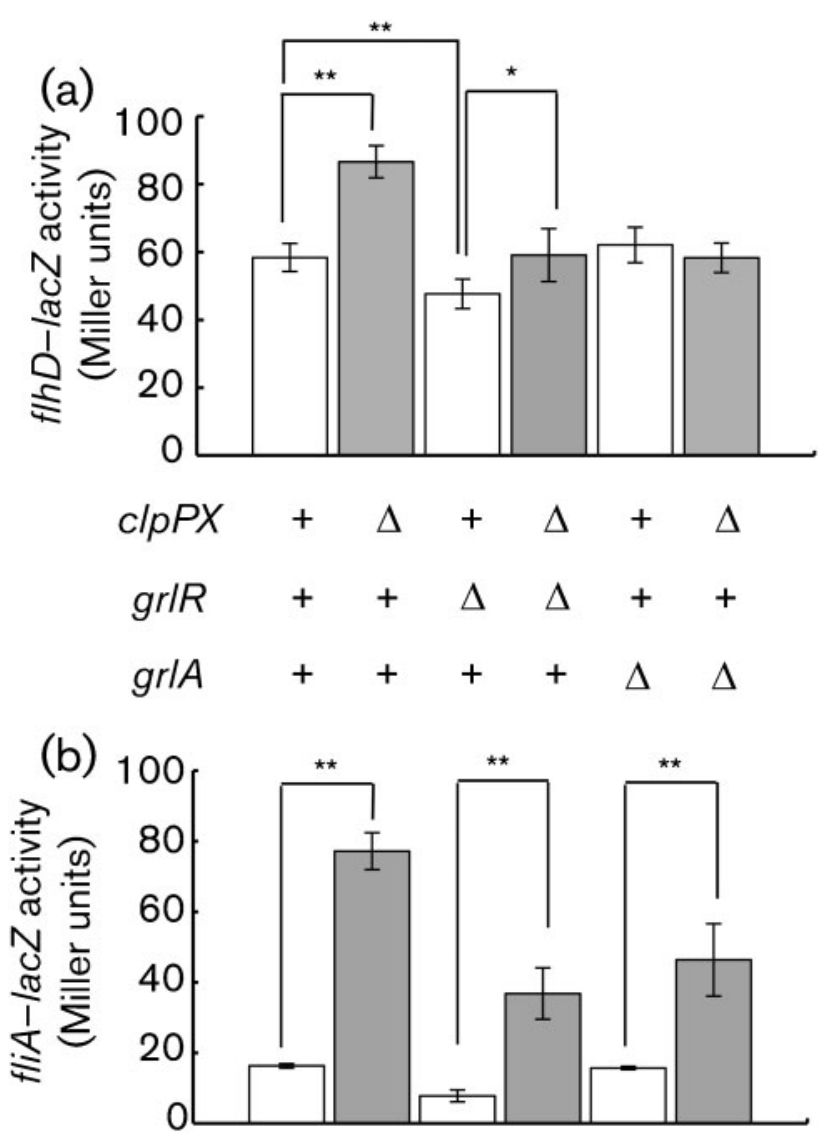

Fig. 4. Effect of depletion of ClpXP protease on the expression of transcriptional $f / h D-l a c Z$ (a) and fliA-lacZ (b) fusions in the absence of $g r / R$ or $g r l A$ in EHEC cells. (a) The expression levels of a chromosomal $f / h D-l a c Z$ fusion were assayed in CS6181 $\left(c / p P X^{+} g_{r l} R^{+} g_{r l} A^{+}\right), \operatorname{CS} 6183\left(\Delta c / p P X g r l R^{+} g r l A^{+}\right)$, CS6320 $\left(c / p P X^{+} \Delta g r l R\right.$ grlA $\left.{ }^{+}\right)$, CS6321 ( $\Delta c / p P X \Delta g r l R$ grlA $\left.{ }^{+}\right)$, CS6322 $\left(c / p P X^{+} g r l R^{+} \Delta g r l A\right)$ and CS6323 $\left(\Delta c / p P X g r l R^{+} \Delta g r l A\right)$. (b) The fliA promoter activities were assayed using PTKY753 carrying a transcriptional fliA-lacZ fusion in CS5693 (clpPX $\left.X^{+} \mathrm{grl}^{+} \mathrm{grl} \mathrm{A}^{+}\right)$, CS5698 ( $\left.\triangle c / p P X ~ g r l R^{+} g_{r l} A^{+}\right)$, CS6281 (clpPX ${ }^{+} \Delta g r l R$ grlA $\left.{ }^{+}\right)$, CS6282 ( $\left.\triangle c / p P X \Delta g r l R g r l A^{+}\right)$, CS6283 (clpPX ${ }^{+}$grlR $\left.{ }^{+} \Delta g r l A\right)$ and CS6284 $\left(\Delta c / p P X g r l R^{+} \Delta g r l A\right)$. Bacterial cells were grown in DMEM for $16 \mathrm{~h}$ at $37^{\circ} \mathrm{C}$. The data are the mean \pm SD for each strain tested in triplicate. ${ }^{*} P<0.05 ;{ }^{* *} P<0.01$.

4a), neither disruption completely abolished the stimulating effect of $c l p P X$ disruption on fliA transcription; the fliA-lacZ transcriptional fusion in grlR and grlA disruption backgrounds was stimulated fourfold (Fig. 4b; compare ${ }^{c l p P} P X^{+}$and $\triangle c l p P X$ in a $\Delta g r l R$ background) and 3.6-fold (compare $c l p P X^{+}$and $\triangle c l p P X$ in a $\triangle g r l A$ background), respectively. Immunoblotting demonstrated that the cellular levels of FliC actually increased after ClpXP depletion in EHEC cells with different grlA and $g r l R$ backgrounds that were grown in DMEM and entered stationary phase (Fig. 5). These results indicate that the regulatory effect of ClpXP on fliA transcription under the LEE-expressing 
condition could depend on not only transcriptional control of the $f l h D C$ operon via the GrlR-GrlA-dependent pathway but also the post-translational control of the FlhD and FlhC proteins.

To determine this possibility, we compared the levels of FlhD and FlhC proteins in $c l p P X^{+}$and $\triangle c l p P X$ cells where $f h D C$ was expressed from the $\mathrm{P}_{\mathrm{AllacO}-1}$ promoter, to exclude the effect of transcriptional control of the flhDC operon. For this purpose, strains with $c l p P X^{+}$and $\triangle c l p P X$ were transformed with the plasmid pTKY802, in which the $f h D C$ promoter had been replaced by the $\mathrm{P}_{\mathrm{AllacO}-1}$ promoter. EHEC cells were harvested when cultures entered stationary phase in DMEM and FlhD and FlhC proteins were detected by immunoblotting. Fig. 6 shows that FlhD and FlhC were detectable in the absence of IPTG, a condition in which $f l h D$ and $f l h C$ were expressed by read-through from the $\mathrm{P}_{\mathrm{AllacO}-1}$ promoter. The results show that disruption of clpPX significantly increased the levels of FlhD and FlhC even when the corresponding gene was expressed by an external promoter system, suggesting that $\mathrm{FlhD}$ and FlhC are controlled post-translationally by ClpXP in EHEC cells under conditions where LEE-encoded GrlA and GrlR are induced.

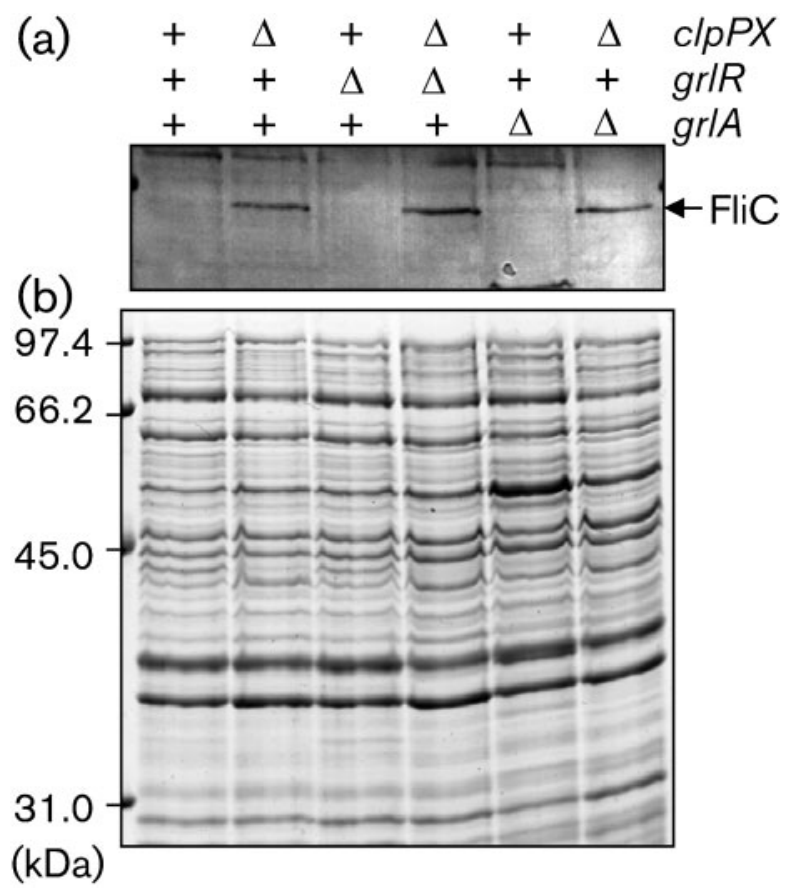

Fig. 5. Cellular levels of FliC protein in EHEC cells with different genetic backgrounds grown in DMEM. The bacterial strains used were CS5295 (clpPX $\left.{ }^{+} \mathrm{grlR}^{+} \mathrm{grlA}^{+}\right), \mathrm{CS5297}\left(\Delta c / p P X \mathrm{grlR} \mathrm{R}^{+}\right.$ $\left.g r l A^{+}\right), \operatorname{CS} 6270\left(c / p P X^{+} \Delta g r l R g r l A^{+}\right), \operatorname{CS} 6271(\Delta c / p P X \Delta g r l R$ $\left.g r l A^{+}\right), \operatorname{CS} 6272\left(c / p P X^{+} g r l R^{+} \Delta g r l A\right)$ and $C S 6273(\Delta c / p P X$ $\left.g r / R^{+} \Delta g r l A\right)$. Cells were grown for $16 \mathrm{~h}$ at $37^{\circ} \mathrm{C}$, collected, lysed and separated by using $12.5 \%$ SDS-PAGE gels. The separated proteins were transferred to a membrane and immunostained with anti-FliC antisera (a). Coomassie brilliant blue-stained gels of the samples used for immunostaining are shown in (b).

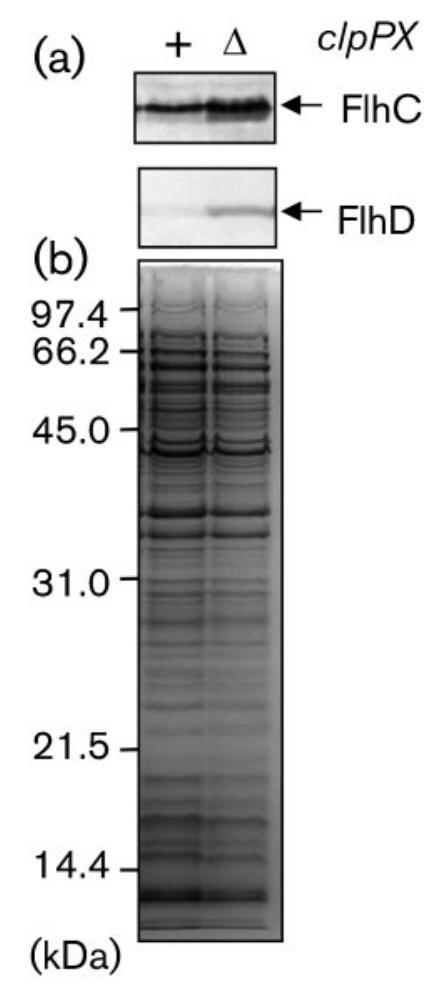

Fig. 6. Cellular levels of $F / h D$ and FlhC proteins by a $P_{A 1-l a c O-1}$ promoter-flhDC fusion in EHEC cells with $c / p P X^{+}$or $\triangle c / p P X$ background that had entered stationary phase in DMEM. Bacterial cells of strain CS5829 $\left(c / p P X^{+}\right)$and CS5830 ( $\left.\triangle c / p P X\right)$ were grown for $16 \mathrm{~h}$ at $37^{\circ} \mathrm{C}$, collected, lysed and separated by using $15 \%$ SDS-PAGE gels. The separated proteins were transferred to a membrane and immunostained with anti-FlhC and anti-FlhD antisera (a). Coomassie brilliant blue-stained gels of the samples used for immunostaining are shown in (b).

These findings suggest that the heightened fliA transcription following ClpXP depletion can be attributed to the additive effects of decreased FlhDC proteolysis and increased flhDC transcription via the GrlR-GrlA-dependent pathway.

\section{DISCUSSION}

We have demonstrated that flagellar gene expression in EHEC is strictly regulated by ClpXP protease through dual pathways (summarized in Fig. 7), i.e. (i) post-translational control of the $\mathrm{FlhD} / \mathrm{FlhC}$ master regulator by degrading them and (ii) transcriptional control of the flhDC operon through the GrlR-GrlA system under conditions in which LEE expression is induced. Concerning the relative importance of ClpXP regulation, it is indicated that post-translational regulation is a greater contributor to the control of flagellar biogenesis than are transcriptional controls. For example, in the analysis of the in vivo stabilities of FlhD and FlhC proteins (Fig. 1b) it would appear that after only $60 \mathrm{~min}, 80 \%$ of FlhD and $90 \%$ of FlhC have been degraded within the cell, while measuring the activities of the flhD-lac $Z$ transcrip- 


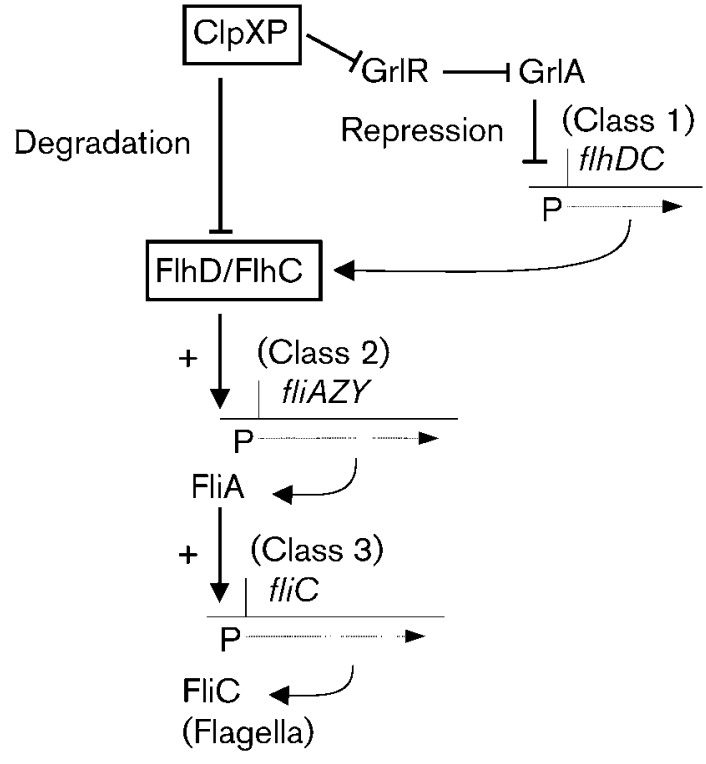

Fig. 7. Diagram of the dual regulatory pathways of flagellar gene expression by ClpXP protease in EHEC. The cellular concentration of $\mathrm{FlhD} / \mathrm{FlhC}$ master regulator, which is essential for transcription of all the genes in the flagellar cascade is tightly controlled at the posttranslational level by ClpXP protease. ClpXP also exerts transcriptional control of the flhDC operon through the GrIR-GrlA system. The role of GrlA as a negative regulator for flhDC transcription and GrIR as an antagonist of GrlA has been demonstrated by lyoda et al. (2006). The present results support ClpXP as the major cause of FlhD/FlhC downregulation via post-translational degradation, but the contribution to transcription is very modest.

tional fusion shows that $f h D$ transcriptional activity increases from $\sim 60$ to 85 Miller units, a modest 1.4-fold increase in the absence of ClpXP (Fig. 4a). We originally demonstrated the tight regulation of flagellar biogenesis due to the control of turnover of FlhD/FlhC proteins by ClpXP in Salmonella (Tomoyasu et al., 2002). Furthermore, we have demonstrated that ClpXP degrades both proteins in the FlhD/FlhC complex but does not seem to recognize the individual subunits (Tomoyasu et al., 2003). Both FlhD and FlhC proteins share high amino acid sequence homology between EHEC (FlhD, NP_310629; FlhC, NP_310628) and Salmonella (FlhD, NP_460882; FlhC, NP_460881). Moreover, ClpX, which is known to be involved in the recognition of protein substrates of the ClpXP protease, is highly conserved in EHEC (NP_308519) and Salmonella (NP_459445). We therefore speculated that both FlhD and FlhC are specifically recognized and degraded by ClpXP in EHEC in manner similar to that in Salmonella.

ClpXP downregulated the transcription of $f l h D C$ in EHEC only when the cells entered stationary phase in DMEM, in which LEE-regulated genes including $g r l R$ and grlA are known to be strongly expressed (Fig. 3). The analysis of transcription efficiency from the $f l h D$ promoter in the $\Delta g r l R$ or $\Delta$ grlA background (Fig. 4) was consistent with regulation through the GrlA-GrlR system, as previously reported, in which ClpXP represses GrlR to antagonize GrlA, which may be repressing the expression of the flhDC operon (Iyoda et al., 2006). Although GrlA has a putative helix-turn-helix DNA-binding motif (Deng et al., 2004), attempts to prove the DNA binding activity of GrlA have been unsuccessful. Therefore, the molecular mechanism by which GrlA represses flhDC transcription remains unknown. How does ClpXP alter the transcription of the flhDC operon? It is unlikely that ClpXP controls the transcriptional activity in a direct manner because of the proteins that form a protease. It is more likely that the effect is indirect, e.g. ClpXP controls the stability of a regulatory protein for the transcription of the $f l h D C$ operon. Iyoda \& Watanabe (2005) studied the possibility that ClpXP may be directly involved in controlling the stability of the GrlR protein by comparing the levels of FLAG-tagged GrlR at different times after arresting protein synthesis in $c l p P X^{+}$and $\triangle c l p P X$ cells. The GrlR-FLAG level in the $c l p P X^{+}$strain was unstable in stationary phase, suggesting that $\mathrm{ClpXP}$ is involved in controlling the stability of GrlR.

A very recent report has demonstrated that flagellar expression is much lower when Salmonella is grown in nutrient-poor medium (MGC) than in rich medium (LB) (Wada et al., 2011). This nutritional control was shown to be executed at a step after flhDC transcription by YdiV, which is enhanced in Salmonella grown in poor medium. The flagellar cascade is known to be very similar in E. coli and Salmonella (Macnab, 1996). However, flagellar expression seems to respond quite differently to nutritional conditions in the two species, though E. coli also possesses $y d i V$ (Zhou et al., 2008). It was anticipated that this difference reflects a difference in $y d i V$ expression (Wada et al., 2011). Although no relationship between increased expression of $y d i V$ and reduced expression of the flagellar genes has yet been established in E. coli, it was assumed that YdiV, if expressed in large enough amounts, could repress flagellar expression.

A study of the function of flagella in the interaction of EHEC O157:H7 with bovine intestinal epithelium has demonstrated that the $\mathrm{H} 7$ flagellum acts as an epithelial adhesion device (Mahajan et al., 2009). Furthermore, its involvement in this crucial step in colonization indicated that H7 flagella could be an important target for intervention strategies. However, in EHEC and enterophathogenic E. coli, most of the bacterial cells attached to the actin pedestals formed on the target cell surfaces were flagellated at an early stage of infection of HeLa cells, while many cells associated with actin pedestals at later stages of infection did not form flagella (Yona-Nadler et al., 2003). Therefore, it is speculated that the GrlR-GrlA system constructed under conditions in which the LEE genes for internalization strategies are expressed could be involved in the downregulation of flagellar formation. Since $\mathrm{H} 7$ flagellins from EHEC O157:H7 are the main triggers inducing expression of the neutrophil chemoattractant interleukin-8 in human intestinal epithelial cells (Berin et al., 2002; Zhou et al., 2003), 
EHEC may need to downregulate flagellar synthesis to evade the host immune responses at a specific stage of infection. In support of this hypothesis, expression of fliC and fliA is repressed during intracellular growth of Salmonella, which depends on functioning of ClpXP (Cummings et al., 2006).

Flagellar synthesis is an energy-consuming process, requiring $2 \%$ of the cell's biosynthetic resources and $0.1 \%$ of the cell's energy is consumed in their rotation (Fontaine et al., 2008). Therefore, flagella are likely to be expressed only when they are needed and their biogenesis should be strictly regulated. The control of turnover of the FlhD/FlhC master regulator by ClpXP could be central to ensuring a limited number of flagella are formed to cope with survival of EHEC, Salmonella and related bacteria in their environments.

\section{REFERENCES}

Abe, H., Tatsuno, I., Tobe, T., Okutani, A. \& Sasakawa, C. (2002). Bicarbonate ion stimulates the expression of locus of enterocyte effacement-encoded genes in enterohemorrhagic Escherichia coli O157:H7. Infect Immun 70, 3500-3509.

Akashi, S., Joh, K., Mori, T., Tsuji, A., Ito, H., Hoshi, H., Hayakawa, T., Ihara, J., Abe, T. \& other authors (1994). A severe outbreak of haemorrhagic colitis and haemolytic uraemic syndrome associated with Escherichia coli O157:H7 in Japan. Eur J Pediatr 153, 650655.

Berin, M. C., Darfeuille-Michaud, A., Egan, L. J., Miyamoto, Y. \& Kagnoff, M. F. (2002). Role of EHEC O157:H7 virulence factors in the activation of intestinal epithelial cell NF- $\kappa \mathrm{B}$ and MAP kinase pathways and the upregulated expression of interleukin 8. Cell Microbiol 4, 635-648.

Bustamante, V. H., Santana, F. J., Calva, E. \& Puente, J. L. (2001). Transcriptional regulation of type III secretion genes in enteropathogenic Escherichia coli: Ler antagonizes H-NS-dependent repression. Mol Microbiol 39, 664-678.

Cherepanov, P. P. \& Wackernagel, W. (1995). Gene disruption in Escherichia coli: $\mathrm{Tc}^{\mathrm{R}}$ and $\mathrm{Km}^{\mathrm{R}}$ cassettes with the option of Flp-catalyzed excision of the antibiotic-resistance determinant. Gene 158, 9-14.

Cummings, L. A., Wilkerson, W. D., Bergsbaken, T. \& Cookson, B. T. (2006). In vivo, fliC expression by Salmonella enterica serovar Typhimurium is heterogeneous, regulated by ClpX, and anatomically restricted. Mol Microbiol 61, 795-809.

Datsenko, K. A. \& Wanner, B. L. (2000). One-step inactivation of chromosomal genes in Escherichia coli K-12 using PCR products. Proc Natl Acad Sci U S A 97, 6640-6645.

Deng, W., Puente, J. L., Gruenheid, S., Li, Y., Vallance, B. A., Vázquez, A., Barba, J., Ibarra, J. A., O'Donnell, P. \& other authors (2004). Dissecting virulence: systematic and functional analyses of a pathogenicity island. Proc Natl Acad Sci U S A 101, 3597-3602.

Donnenberg, M. S. \& Kaper, J. B. (1991). Construction of an eae deletion mutant of enteropathogenic Escherichia coli by using a positive-selection suicide vector. Infect Immun 59, 4310-4317.

Fontaine, F., Stewart, E. J., Lindner, A. B. \& Taddei, F. (2008). Mutations in two global regulators lower individual mortality in Escherichia coli. Mol Microbiol 67, 2-14.

Francez-Charlot, A., Laugel, B., Van Gemert, A., Dubarry, N., Wiorowski, F., Castanié-Cornet, M. P., Gutierrez, C. \& Cam, K. (2003). RcsCDB His-Asp phosphorelay system negatively regulates the flhDC operon in Escherichia coli. Mol Microbiol 49, 823-832.
Friedberg, D., Umanski, T., Fang, Y. \& Rosenshine, I. (1999). Hierarchy in the expression of the locus of enterocyte effacement genes of enteropathogenic Escherichia coli. Mol Microbiol 34, 941-952.

Gamer, J., Bujard, H. \& Bukau, B. (1992). Physical interaction between heat shock proteins DnaK, DnaJ, and GrpE and the bacterial heat shock transcription factor $\sigma 32$. Cell 69, 833-842.

Gottesman, S., Roche, E., Zhou, Y. \& Sauer, R. T. (1998). The ClpXP and ClpAP proteases degrade proteins with carboxy-terminal peptide tails added by the SsrA-tagging system. Genes Dev 12, 1338-1347.

Grant, A. J., Farris, M., Alefounder, P., Williams, P. H., Woodward, M. J. \& O'Connor, C. D. (2003). Co-ordination of pathogenicity island expression by the BipA GTPase in enteropathogenic Escherichia coli (EPEC). Mol Microbiol 48, 507-521.

Habdas, B. J., Smart, J., Kaper, J. B. \& Sperandio, V. (2010). The LysR-type transcriptional regulator QseD alters type three secretion in enterohemorrhagic Escherichia coli and motility in K-12 Escherichia coli. J Bacteriol 192, 3699-3712.

lyoda, S. \& Watanabe, H. (2005). ClpXP protease controls expression of the type III protein secretion system through regulation of RpoS and GrlR levels in enterohemorrhagic Escherichia coli. J Bacteriol 187, 4086-4094.

lyoda, S., Koizumi, N., Satou, H., Lu, Y., Saitoh, T., Ohnishi, M. \& Watanabe, H. (2006). The GrlR-GrlA regulatory system coordinately controls the expression of flagellar and LEE-encoded type III protein secretion systems in enterohemorrhagic Escherichia coli. J Bacteriol 188, 5682-5692.

Lehnen, D., Blumer, C., Polen, T., Wackwitz, B., Wendisch, V. F. \& Unden, G. (2002). LrhA as a new transcriptional key regulator of flagella, motility and chemotaxis genes in Escherichia coli. Mol Microbiol 45, 521-532.

Macnab, R. M. (1996). Flagella and motility. In Escherichia coli and Salmonella: Cellular and Molecular Biology, 2nd edn, pp. 123-145. Edited by F. C. Neidhardt, R., III, H. L. Ingraham, E. C. C. Lin, K. B. Low, B. Magasanik, W. S. Reznikoff, M. Riley, M. Schaechter \& H. E. Umbarger. Washington, D.C.: American Society for Microbiology.

Macnab, R. M. (2003). How bacteria assemble flagella. Annu Rev Microbiol 57, 77-100.

Mahajan, A., Currie, C. G., Mackie, S., Tree, J., McAteer, S., McKendrick, I., McNeilly, T. N., Roe, A., La Ragione, R. M. \& other authors (2009). An investigation of the expression and adhesin function of $\mathrm{H} 7$ flagella in the interaction of Escherichia coli O157 : H7 with bovine intestinal epithelium. Cell Microbiol 11, 121-137.

Ortega, J., Singh, S. K., Ishikawa, T., Maurizi, M. R. \& Steven, A. C. (2000). Visualization of substrate binding and translocation by the ATP-dependent protease, ClpXP. Mol Cell 6, 1515-1521.

Schneider, K. \& Beck, C. F. (1986). Promoter-probe vectors for the analysis of divergently arranged promoters. Gene 42, 37-48.

Shi, W., Li, C., Louise, C. J. \& Adler, J. (1993). Mechanism of adverse conditions causing lack of flagella in Escherichia coli. J Bacteriol 175, 2236-2240.

Shin, S. \& Park, C. (1995). Modulation of flagellar expression in Escherichia coli by acetyl phosphate and the osmoregulator OmpR. J Bacteriol 177, 4696-4702.

Soutourina, O., Kolb, A., Krin, E., Laurent-Winter, C., Rimsky, S., Danchin, A. \& Bertin, P. (1999). Multiple control of flagellum biosynthesis in Escherichia coli: role of H-NS protein and the cyclic AMP-catabolite activator protein complex in transcription of the flhDC master operon. J Bacteriol 181, 7500-7508.

Soutourina, O. A., Krin, E., Laurent-Winter, C., Hommais, F., Danchin, A. \& Bertin, P. N. (2002). Regulation of bacterial motility in response to low $\mathrm{pH}$ in Escherichia coli: the role of H-NS protein. Microbiology 148, 15431551. 
Sperandio, V., Torres, A. G. \& Kaper, J. B. (2002). Quorum sensing Escherichia coli regulators B and C (QseBC): a novel two-component regulatory system involved in the regulation of flagella and motility by quorum sensing in E. coli. Mol Microbiol 43, 809-821.

Takaya, A., Matsui, M., Tomoyasu, T., Kaya, M. \& Yamamoto, T. (2006). The DnaK chaperone machinery converts the native $\mathrm{FlhD}_{2} \mathrm{C}_{2}$ hetero-tetramer into a functional transcriptional regulator of flagellar regulon expression in Salmonella. Mol Microbiol 59, 1327-1340.

Tomoyasu, T., Ohkishi, T., Ukyo, Y., Tokumitsu, A., Takaya, A., Suzuki, M., Sekiya, K., Matsui, H., Kutsukake, K. \& Yamamoto, T. (2002). The ClpXP ATP-dependent protease regulates flagellum synthesis in Salmonella enterica serovar typhimurium. J Bacteriol 184, 645-653.

Tomoyasu, T., Takaya, A., Isogai, E. \& Yamamoto, T. (2003). Turnover of FlhD and FlhC, master regulator proteins for Salmonella flagellum biogenesis, by the ATP-dependent ClpXP protease. Mol Microbiol 48, 443-452.

Tomoyasu, T., Takaya, A., Handa, Y., Karata, K. \& Yamamoto, T. (2005). ClpXP controls the expression of LEE genes in enterohaemorrhagic Escherichia coli. FEMS Microbiol Lett 253, 59-66.

Umanski, T., Rosenshine, I. \& Friedberg, D. (2002). Thermoregulated expression of virulence genes in enteropathogenic Escherichia coli. Microbiology 148, 2735-2744.

Vazquez-Juarez, R. C., Kuriakose, J. A., Rasko, D. A., Ritchie, J. M., Kendall, M. M., Slater, T. M., Sinha, M., Luxon, B. A., Popov, V. L. \& other authors (2008). CadA negatively regulates Escherichia coli O157: H7 adherence and intestinal colonization. Infect Immun 76, 5072-5081.
Wada, T., Morizane, T., Abo, T., Tominaga, A., Inoue-Tanaka, K. \& Kutsukake, K. (2011). EAL domain protein YdiV acts as an anti-FlhD ${ }_{4} \mathrm{C}_{2}$ factor responsible for nutritional control of the flagellar regulon in Salmonella enterica serovar Typhimurium. J Bacteriol 193, 16001611.

Wei, B. L., Brun-Zinkernagel, A. M., Simecka, J. W., Prüß, B. M., Babitzke, P. \& Romeo, T. (2001). Positive regulation of motility and flhDC expression by the RNA-binding protein CsrA of Escherichia coli. Mol Microbiol 40, 245-256.

Wozniak, C. E., Lee, C. \& Hughes, K. T. (2009). T-POP array identifies EcnR and PefI-SrgD as novel regulators of flagellar gene expression. J Bacteriol 191, 1498-1508.

Yona-Nadler, C., Umanski, T., Aizawa, S., Friedberg, D. \& Rosenshine, I. (2003). Integration host factor (IHF) mediates repression of flagella in enteropathogenic and enterohaemorrhagic Escherichia coli. Microbiology 149, 877-884.

Zhou, Y., Gottesman, S., Hoskins, J. R., Maurizi, M. R. \& Wickner, S. (2001). The RssB response regulator directly targets $\sigma^{\mathrm{S}}$ for degradation by ClpXP. Genes Dev 15, 627-637.

Zhou, X., Girón, J. A., Torres, A. G., Crawford, J. A., Negrete, E., Vogel, S. N. \& Kaper, J. B. (2003). Flagellin of enteropathogenic Escherichia coli stimulates interleukin-8 production in T84 cells. Infect Immun 71, 2120-2129.

Zhou, X., Meng, X. \& Sun, B. (2008). An EAL domain protein and cyclic AMP contribute to the interaction between the two quorum sensing systems in Escherichia coli. Cell Res 18, 937-948.

Edited by: A. H. M. van Vliet 